\title{
End-stage Renal Disease and Diabetes Catalyze the Formation of a Pentose-derived Crosslink from Aging Human Collagen
}

David R. Sell and Vincent M. Monnier

Institute of Pathology, Case Western Reserve University, School of Medicine, Cleveland, Ohio 44106

\section{Abstract}

Structure elucidation of a specific fluorophore from the aging extracellular matrix revealed the presence of a protein crosslink formed through nonenzymatic glycosylation of lysine and arginine residues. The unexpected finding that a pentose instead of a hexose is involved in the crosslinking process suggested that the crosslink, named pentosidine, might provide insight into abnormalities of pentose metabolism in aging and disease. This hypothesis was investigated by quantitating pentosidine in hydrolysates of $\mathbf{1 0 3}$ human skin specimens obtained randomly at autopsy.

Pentosidine level was found to increase exponentially from 5 to $75 \mathrm{pmol} / \mathrm{mg}$ collagen over lifespan $(r=0.86, P<0.001)$. A three- to tenfold increase was noted in insulin-dependent diabetic and nondiabetic subjects with severe end-stage renal disease requiring hemodialysis $(P<0.001)$. Moderately elevated levels were also noted in some very old subjects, some subjects with non-insulin dependent diabetes, and two subjects with cystic fibrosis and diabetes. The cause of the abnormal pentose metabolism in these conditions is unknown but may relate to hemolysis, impaired pentose excretion, cellular stress, and accelerated breakdown of ribonucleotides.

Thus, pentosidine emerges as a useful tool for assessment of previously unrecognized disorders of pentose metabolism in aging and disease. Its presence in red blood cells and plasma proteins suggests that it might be used as a measure of integrated pentosemia in analogy to glycohemoglobin for the assessment of cumulative glycemia. (J. Clin. Invest. 1990. 85:380-384.) advanced glycosylation • arginine $\bullet$ imidazopyridine $\bullet$ lysine $\bullet$ Maillard reaction

\section{Introduction}

Recently this laboratory reported the presence of skin collagen-linked fluorescence that accumulates during normal aging and that was increased in $98 \%$ of insulin-dependent diabetic subjects (1). There was an overall correlation between collagen-linked fluorescence and severity of retinopathy, arterial and joint stiffness, and systolic blood pressure. The analogy between the fluorescence properties of diabetic skin and those of collagen incubated with glucose suggested that advanced glycosylation (Maillard) reaction had formed at a higher rate in

Address correspondence to Dr. Vincent $\mathrm{M}$. Monnier, Institute of $\mathrm{Pa}$ thology, Case Western Reserve University, 2085 Adelbert Road, Cleveland, $\mathrm{OH} 44106$.

Received for publication 8 January 1988 and in revised form 28 July 1989.

J. Clin. Invest.

(c) The American Society for Clinical Investigation, Inc.

0021-9738/90/02/0380/05 \$2.00

Volume 85, February 1990, 380-384 diabetes, presumably reflecting chronic elevation of glycemia over many years.

Work was initiated to substantiate the glucose-derived nature of the fluorescence. Human insoluble collagen was fractionated into low molecular weight peptides and two distinct fluorescent molecules were obtained (2). The fluorophore that was increased in $98 \%$ of diabetic subjects was unfortunately labile to acid hydrolysis and the involvement of glucose in its structure remains to be confirmed. However, the second fluorophore with excitation-emission maxima at 335 and 385 $\mathrm{nm}$, respectively, was resistant to acid hydrolysis, and its structure could be elucidated and verified by total synthesis (3). It revealed the presence of a crosslink between L-lysine and L-arginine residues that could be synthesized by ribose (Fig. 1). Because other pentoses could also serve as precursors of the crosslink, this advanced glycosylation endproduct was named pentosidine. The overall order of reactivity of pentoses in the synthesis of pentosidine with collagen followed the sequence ribose $\gg$ lyxose $>$ arabinose $\sim$ xylose. Hexoses and pentuloses like xylulose and ribulose were unable to produce the crosslink.

The involvement of a pentose in the crosslink was unexpected for two reasons. First, total plasma pentose concentration in the human is 100 times less than that of glucose, an observation that may have evolutionary significance since rodents have very high ribose concentrations (4) and a short lifespan that could be related to accelerated ribose-mediated crosslinking. Second, a survey of the literature revealed that, aside from essential pentosuria, abnormalities of pentose metabolism were virtually unexplored except for rare and remote studies on ribose disposal rates in diabetes $(5,6)$.

The availability of pentosidine that can be quantitated in tissues at the picomole level suggested that it could be useful for the clinical assessment of impaired pentose metabolism, if indeed such abnormality exists. To investigate this hypothesis, a pilot study on pentosidine level in skin obtained at autopsy was performed. The results, reported below, were statistically correlated with diagnoses obtained from postmortem records.

\section{Methods}

A total of 103 human skin samples were obtained from a collection of tissue acquired by our laboratory at autopsy and currently stored at $-80^{\circ} \mathrm{C}$ by autopsy number. Tissues were randomly selected from the collection to include a broad age range. Additional tissues from subjects with type I and II diabetes were provided by the National Disease Research Interchange (Philadelphia, PA). The final selection comprised 56 males, 47 females, $64 \%$ caucasian, and $36 \%$ black individuals. Autopsy records were obtained for all subjects. For each individual, a list of the major anatomical and clinical diagnoses was compiled. Diagnoses were computer coded and graded from absent to severe depending on the clinical and anatomical assessment on the autopsy report. Diagnoses and assessment ratings were as follows: diabetes, 0 (not present), 1 (type I), 2 (type II); chronic renal failure, 0 (absent), 1 
<smiles></smiles>

Figure 1. Structure of pentosidine.

(moderate), 2 (severe, requiring hemodialysis and/or renal transplant); nephrosclerosis, 0 (absent), 1 (present); systemic and coronary atherosclerosis, 0 (not visually detectable), 1 (mild), 2 (moderate), 3 (severe); hypertension, 0 (absent), 1 (present).

The data on nondiabetic and diabetic subjects are summarized in Table I. The diagnosis of diabetes was based on information available from the chart.

Preparation of skin collagen for HPLC assay. Insoluble collagen was prepared from skin samples as previously described (1). The collagen samples ( $25 \mathrm{mg}$ blotted wet weight) were acid-hydrolyzed in $2 \mathrm{ml}$ of $6 \mathrm{~N}$ hydrochloric acid for $24 \mathrm{~h}$ at $110^{\circ} \mathrm{C}$. The acid was evaporated and each sample was reconstituted in $1 \mathrm{ml}$ of water containing $0.01 \mathrm{M}$ heptafluorobutyric acid (HFBA). ${ }^{1}$ Collagen content was determined for all samples and its concentration was adjusted to equal amounts before analysis by HPLC.

HPLC assay for pentosidine in skin. Pentosidine was determined in acid-hydrolyzed skin samples by reverse-phase HPLC. Samples of 36 $\mu$ l, equivalent to $256 \mu \mathrm{g}$ of collagen, were injected onto an HPLC (Millipore Corp., Waters Associates, Milford, MA) equipped with model 510 pumps, a U6K manual injector, and a model 680 controller. The effluent from the HPLC was directed onto an on-line Fluorichrom fluorescence detector (model 03-905831-00; Varian Associates, Inc., Palo Alto, CA) containing a 334-nm interference excitation filter and a type 4-76 broad band (340-640 nm) emission filter. The detector controls were set as follows: lamp intensity, low; photomultiplier gain, high; and attenuator position at number 5 . Chromatographs were recorded with a strip chart recorder (model D5217-5AQ; Houston Instrument, Inc., Austin, TX) with a chart speed of 12.5 $\mathrm{cm} / 60 \mathrm{~min}$ and attenuator switch at $1 \mathrm{mV}$ full scale. Separations were made on a 4.6-mm $\times 25-\mathrm{cm}$ Vydac type $218 \mathrm{TP}(10 \mu \mathrm{m}) \mathrm{C}-18$ column (The Separations Group, Hesperia, CA) with a linear gradient program of $10-17 \%$ acetonitrile from 0 to $35 \mathrm{~min}$ containing water and $0.01 \mathrm{M}$ HFBA as counterion. Since not all samples could be analyzed on the same day, a calibrator consisting of $36 \mu \mathrm{l}$ of a $1.23-\mathrm{nmol}$ pyridoxamine standard (Sigma Chemical Co., St. Louis, MO) was used. However, daily fluctuations of the fluorometer were found to be minimal, and data did not need to be corrected for this factor. A representative chromatogram of pentosidine in skin is shown in Fig. 2. Quantitation was performed by comparison of peak area of unknowns to that of a purified pentosidine standard. Results were expressed as picomoles of synthetic pentosidine per milligram of collagen.

Assay for collagen. Collagen was quantitated by assaying for hydroxyproline by the method of Stegeman and Stalder (7). The amount of collagen was estimated assuming a content of $14 \%$ hydroxyproline by weight (8).

Statistical analysis. Normality and variance equalities were tested according to the procedures of Shapiro and Wilk (9) and Steel and Torrie (10), respectively. Data were transformed by use of the square root transformation according to Steel and Torrie (10). Simple and multiple regression techniques were from Neter and Wasserman (11) with use of methods available in the SPSS/PC+ statistical software (12). Confidence interval for the regression line was computed using the error of prediction formula given by Armitage (13). The Wilcoxon-Mann-Whitney two-sample test was from Steel and Torrie (10).

1. Abbreviations used in this paper: ESR, end-stage renal disease; HFBA, heptafluorobutyric acid.
For this test, observations from two groups were ranked from smallest to largest. A " $Z$ " statistic was computed from given formulas and significance determined by a normal distributions function table (10).

\section{Results}

The effect of age on pentosidine level in skin of individuals without diabetes or renal failure is shown in Fig. 3. A curvilinear, exponential increase was noted with age that corresponded to a sixfold increase between 10 and $100 \mathrm{yr}$; however, beyond the age of $80 \mathrm{yr}$ pentosidine values started to spread, some being very high while others remained low. The three highest values were found in subjects with no apparent metabolic disease who died of congestive heart failure (age 85) and sepsis (ages 84 and 89 ), respectively. Surprisingly, the one individual with a level below the confidence interval died from a ruptured aortic aneurysm associated with severe atherosclerosis.

The relationship between age, diabetes, renal disease, and pentosidine level in control subjects is shown in Fig. 4. In further refinement, the effect of age was removed and subjects with renal disease were reclassified for severity (Fig. 5). The highest levels were noted for nondiabetic and diabetic individuals with end-stage renal disease (ESR) requiring hemodialysis and/or renal transplant. Renal failure without ESR, however, did not cause elevation of pentosidine in nondiabetic or noninsulin dependent diabetic subjects (Figs. 4 and 5).

Moderately elevated levels were found in four individuals with type I diabetes who had no overt renal complications (Fig. 4). Two died from complications of cystic fibrosis (ages 21 and 33 ), one from trauma (age 28), and one from complications of bypass surgery (age 44 ). This latter hypertensive individual had diabetes for $24 \mathrm{yr}$ and a smoking history of $30 \mathrm{yr}$ associated with complications of severe coronary and systemic atherosclerosis, but apparently no renal or retinal problems.

Pentosidine level in subjects with type II diabetes without renal failure exhibited no set pattern with regard to duration of diabetes, treatment, or complications. Four individuals had pentosidine levels on or above the confidence interval. One had diabetes for $1 \mathrm{yr}$ and died from prostatic cancer (age 70), and the other three had diabetes for 5 (age 42), 8 (age 59), and $10 \mathrm{yr}$ (age 83) and died from congestive heart failure associated with atherosclerotic cardiovascular disease.

Of the six individuals with type II diabetes with chronic renal insufficiency, pentosidine levels were normal in three subjects (ages 68, 75, and 85 ) with mild renal failure. Elevated pentosidine levels were found in two subjects with ESR requiring hemodialysis (ages 46 and 61). A striking observation was that all diabetic individuals with renal failure and levels outside the confidence interval had retinopathy implicitly mentioned in their postmortem record, while no mention of this diagnosis was made for subjects with levels found within the confidence interval for control subjects.

As noted above, severity of renal failure appeared to play a role in explaining the increase in pentosidine in nondiabetic individuals as well. Subjects with mild chronic renal insuffciency had levels within the confidence interval. In all three subjects with ESR requiring hemodialysis, elevated pentosidine levels were observed (Figs. 4 and 5). The 29-yr-old subject with moderate elevation of pentosidine (Fig. 4) had been on dialysis for 19 mo before renal transplant, which he survived for $20 \mathrm{~d}$. The other two individuals were on dialysis for $3 \mathrm{yr}$ 
Table I. Summary of Patient Data

\begin{tabular}{|c|c|c|}
\hline Category & No. of subjects & Major diagnoses \\
\hline \multicolumn{3}{|l|}{ Nondiabetic } \\
\hline$<80 \mathrm{yr}$ & 47 & $\begin{array}{l}\text { Congenital heart disease (6), atherosclerotic cardiovascular disease with congestive heart } \\
\text { failure (4), leukemia (5), cancer (19), cirrhosis of liver (3), all others (10) }\end{array}$ \\
\hline$>80 \mathrm{yr}$ & 13 & Atherosclerotic cardiovascular disease with congestive heart failure (6) \\
\hline $\begin{array}{l}\text { With mild } \\
\text { renal failure }\end{array}$ & 6 & $\begin{array}{l}\text { Atherosclerotic cardiovascular disease with congestive heart failure (4), polyarteritis nodosa } \\
\text { (1), lupus erythematosus (1) }\end{array}$ \\
\hline With ESR & 3 & $\begin{array}{l}\text { Atherosclerotic cardiovascular disease with congestive heart failure (2), post-streptococcal } \\
\text { glomerulonephritis (1) (hemodialysis [3], renal transplant [1]) }\end{array}$ \\
\hline \multicolumn{3}{|r|}{ - } \\
\hline $\begin{array}{l}\text { Type I without } \\
\text { renal failure* }\end{array}$ & 4 & $\begin{array}{l}\text { Atherosclerotic cardiovascular disease with congestive heart failure (1), cystic fibrosis (2), } \\
\text { trauma (1) }\end{array}$ \\
\hline Type I with ESR & 7 & $\begin{array}{l}\text { ESR (7) (hemodialysis [5], renal transplant [3], retinopathy [7], atherosclerotic cardiovascular } \\
\text { disease [4], neuropathy [4], gangrene [1]) }\end{array}$ \\
\hline $\begin{array}{l}\text { Type II without } \\
\text { renal failure }\end{array}$ & 17 & $\begin{array}{l}\text { Congestive heart failure (13), cancer (1), meningitis (1), leukemia (1), pneumonia (1) } \\
\text { (atherosclerotic cardiovascular disease [17], gangrene [3]) }\end{array}$ \\
\hline $\begin{array}{l}\text { Type II with mild } \\
\text { renal failure }\end{array}$ & 3 & Congestive heart failure (2), cancer (1) (atherosclerotic cardiovascular disease [3], gangrene [1]) \\
\hline Type II with ESR & 3 & $\begin{array}{l}\text { Atherosclerotic cardiovascular disease with congestive heart failure (3) (hemodialysis [2], } \\
\text { retinopathy [2], gangrene [2]) }\end{array}$ \\
\hline
\end{tabular}

Primary diagnoses with further complications as given in brackets. * Two subjects afflicted with cystic fibrosis and diabetes have been placed in this group by convenience, but are not type I diabetics as defined (25).

before death due to heart failure associated with severe atherosclerotic heart disease.

For statistical evaluation, pentosidine levels were adjusted for age and classified into nine different groups (Fig. 5). Due to large differences in the range of pentosidine level within groups, means were compared by a nonparametric statistic rather than by analysis of variance, and a multiple comparison test because variance was not homogenous among groups even with statistical transformation. Results confirmed the visual impression that mean pentosidine level for nondiabetic controls was significantly less than for the following groups: nondiabetic individuals with ESR, $P<0.002$; type I diabetic subjects without renal failure, $P<0.001$, and with ESR, $P$ $<0.0001$. Mean pentosidine level was not significantly elevated in subjects with type II diabetes unless ESR was present $(P<0.004)$.

Multivariate analysis was used to evaluate the correlation between pentosidine level, age, race, sex, presence and type of diabetes, severity of renal failure, atherosclerosis, nephrosclerosis, and hypertension. Age $(P<0.001)$ and renal failure $(P$

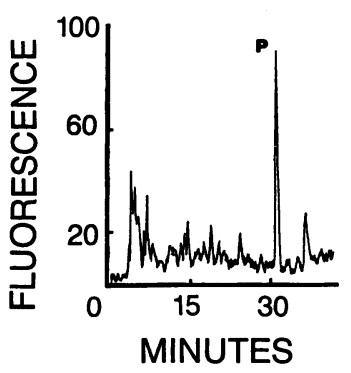

Figure 2. Reverse-phase HPLC analysis of pentosidine in acid-hydrolyzed human skin collagen. The sample was eluted with a linear gradient of $10-17 \%$ acetonitrile from 0 to $35 \mathrm{~min}$ containing water and $0.01 \mathrm{M}$ HFBA. A total of $256 \mu \mathrm{g}$ of collagen contained in $36 \mu$ l of water $/ 0.01 \mathrm{M}$ HFBA was injected onto the HPLC. The effluent was monitored by a fluorescence detector containing a 334$\mathrm{nm}$ interference excitation filter and a broad band (340-640 $\mathrm{nm}$ ) emission filter. Pentosidine $(P)$ eluted at $31 \mathrm{~min}$.
$<0.001$ ) were selected after stepwise multiple regression analysis with $r^{2}=0.56$ and the functional relation $Y=X^{2}$, where $Y$ $=$ pentosidine level (picomoles/milligram collagen) and $X$ $=2.95+0.036$ (age) +1.51 (renal failure). Diabetes became

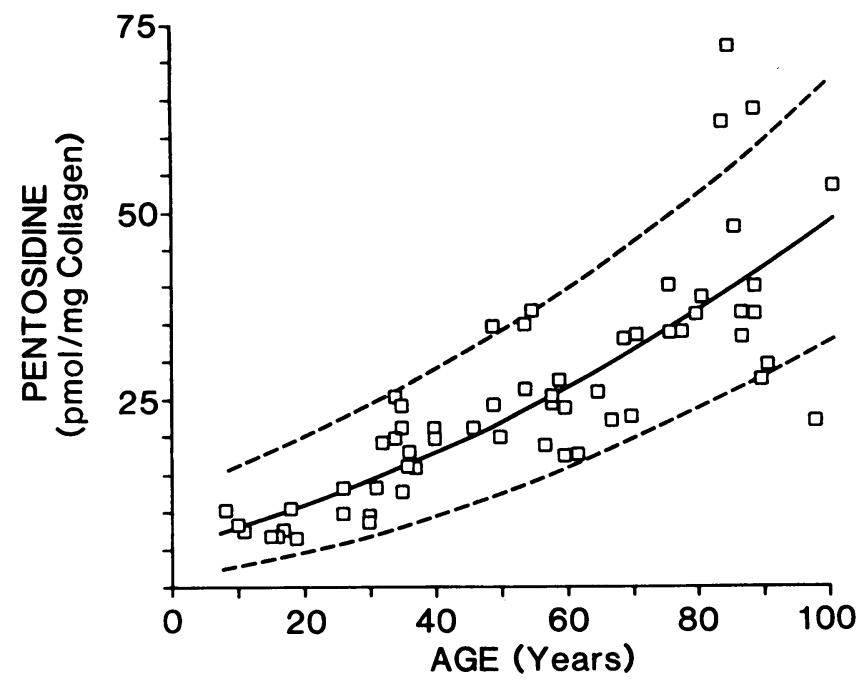

Figure 3. Pentosidine levels expressed as a function of age for nondiabetic subjects without renal failure (Table I), together with a regression line and a $95 \%$ confidence interval. An age-dependent variation was noted which was particularly large for ages $>80 \mathrm{yr}$. Therefore, in computing the regression line, data points from ages 8-80 were used, which were on the part of the plot exhibiting less variability. For statistical computation and to further stabilize the variation, all data were initially transformed using the square root transformation $(10$, 11 ). The asymmetry of the confidence interval is due to the presentation of the data in the untransformed form. $r=0.86$. Regression line equation: $y=0.002 X^{2}+0.214 X+5.69$. 


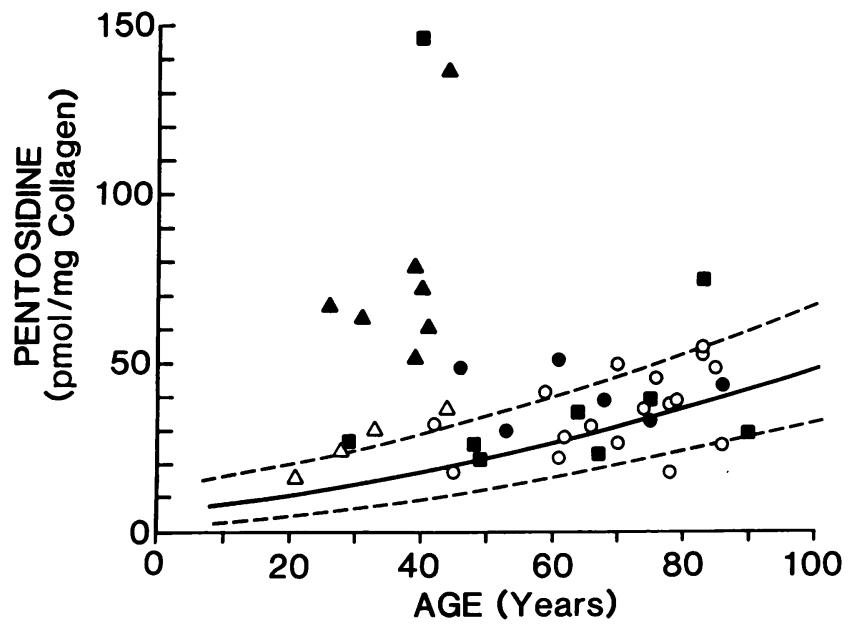

Figure 4. The relationship between pentosidine and the presence of diabetes or renal failure. Levels are expressed relative to a normal range computed as a $95 \%$ confidence interval determined and reproduced from the regression line of Fig. 3. Subjects without renal failure are represented by open symbols, while subjects with renal failure are represented by closed symbols. $n$, Nondiabetic with renal failure; $\Delta$, type I diabetic without renal failure; $\Delta$, type I diabetic with renal failure; $\bigcirc$, type II diabetic without renal failure; $\bullet$ type II diabetics with renal failure.

significant $(P<0.034)$ only when subjects were reclassified for the presence or absence of diabetes without regard to type of insulin dependency $\left(r^{2}=0.58\right)$.

\section{Discussion}

The results of pentosidine measurement in human skin collagen suggests the presence of a profound abnormality of pentose metabolism in subjects with ESR requiring hemodialysis. The abnormality seems to be accentuated by diabetes and also to be present to a milder degree in some diabetic subjects that

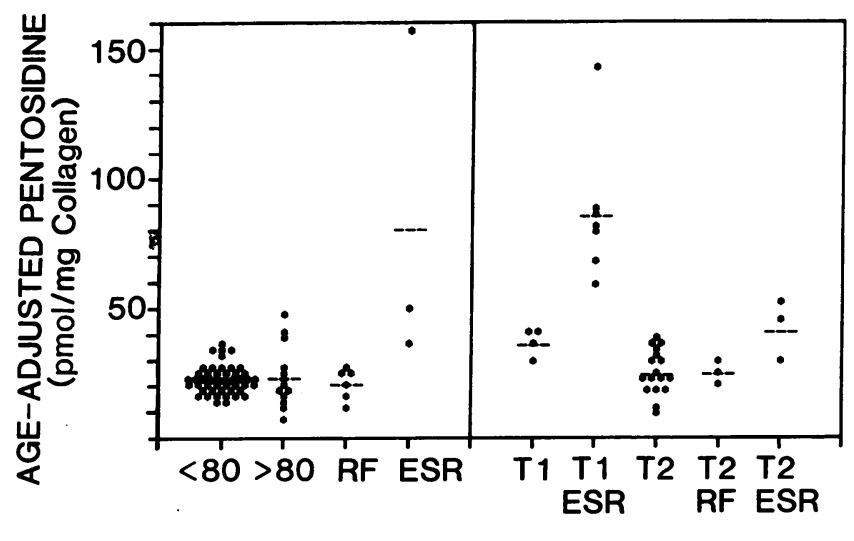

\section{NONDIABETIC}

DIABETIC

Figure 5. The effects of old age, diabetes, and renal failure on pentosidine level. Levels were normalized to the age of 50 for all subjects, expressed relative to a normal control group $(<80)$. $<80$, Ages 8-80 without renal failure; $>80$, ages $>80$ without renal failure; $R F$, mild renal failure; $E S R$, end-stage renal disease requiring hemodialysis and/or renal transplants; $T 1$, type I diabetes; $T 2$, type II diabetes; ---, mean. are not in renal failure. Finally, rapid increase in pentosidine level seems to occur in some subjects reaching the end of lifespan. These observations raise important questions concerning the etiology and consequences of the impaired pentose metabolism as reflected by pentosidine.

The nature of the pentose involved in pentosidine synthesis in vivo is unknown. In vitro, ribose is two to three times more reactive than arabinose and xylose, suggesting that ribose might be the major precursor of pentosidine in vivo. On the other hand, these sugars are excreted in the urine with excretion rates of free arabinose, xylose, and ribose reported to be $14,8.5$, and $5 \mu \mathrm{g} / \mathrm{min}$, respectively (14). Thus, it is possible that decreased clearance rate by the diseased kidney would explain increased pentosidine formation in uremia. The presence of arabinose and xylose in the plasma of nonuremic individuals is thought to result primarily from dietary sources, whereas that of ribose is thought to result from endogenous sources $(15,16)$.

Although the source of free ribose is unclear, one could speculate that uremia induces a toxic effect onto cells, which leads to a catabolic state with decreased ribose tolerance, increased ribonucleotide breakdown, and decreased reutilization of ribose or its metabolites. In this regard, the detection of large quantities of pentosidine in fibroblasts or glomerular mesangial cells contained in cell culture (3) strongly favors this proposition. For this reason, it is tempting to propose that pentosidine formation might be a measure of chronic cellular stress. A third possible source of free pentose could come from chronic hemolysis and dephosphorylation of ribose-5-phosphate which is formed by the pentose shunt. Ribose-5-phosphate itself, however, is not a precursor of pentosidine (3). Clarification of the type and source of the pentose involved in pentosidine formation will require experimental determination of each individual pentose in the plasma. Surprisingly, these data are not available in the literature. This may relate to the difficulty of quantitating pentoses and the lack of an incentive for such measurements until now.

The biological consequences of increased pentose-mediated crosslinking in ESR can be modeled according to those attributed to the advanced glycosylation reaction in diabetes (17). In effect, the complications of chronic renal failure resemble those of diabetes, both being characterized by accelerated cardiovascular disease (18), microangiopathy and basement membrane thickening (19), impaired T cell function (20, 21), cataracts, and peripheral neuropathy, to mention just a few (22). Thus, it is tempting to speculate that the dramatic increase in pentose-derived advanced glycosylation endproducts might be in part responsible for the pathogenesis of the chronic complications of ESR.

One additional observation of the potential relevance to the role of pentose-mediated molecular damage in ESR is the presence of so-called uremic middle molecules in the blood of uremic patients. These middle molecules include guanidinoacetic acid and methylguanidine $(23,24)$, both of which contain a guanidino group that can compete with arginine (unpublished results) and thus presumably prevent pentosidine formation in vivo. Thus, these middle molecules would act like aminoguanidine, which is currently under evaluation as an inhibitor of the advanced glycosylation reaction in diabetes (17). Their accumulation in the blood of uremic patients would thus constitute a sort of safety mechanism, though overwhelmed, for the trapping of highly toxic dicarbonyl 
compounds derived from sugars and other sources. This new interpretation of the significance of middle molecules in uremia is contrary to the prevailing assumption that middle molecules are uremic toxins (24).

Although abnormalities of pentose metabolism were most dramatically revealed by pentosidine in uremic subjects, more moderate but significant abnormalities were also apparent in very old age and in some diabetic individuals. The observation that all diabetic subjects with elevated pentosidine levels had retinopathy may signal a preexisting subtle impairment of pentose metabolism predictive for the risk of developing microvascular disease under hyperglycemic stress. A systematic investigation of that question is indicated.

Because pentosidine was also detected in human red blood cells and plasma proteins ( 3 ) it is likely that the abnormalities described above and others yet to be uncovered could be assessed by using a blood-based instead of a skin-biopsy assay for pentosidine. Its presence in red blood cells is expected to provide information on cumulative pentosemia in analogy to the information provided by glycohemoglobin on cumulative glycemia. These considerations suggest that pentosidine might become a prime tool for the assessment and management of disorders of pentose metabolism.

\section{Acknowledgments}

We thank the National Disease Research Interchange, Philadelphia, for providing human tissues.

This work was supported by grants AG-05601, AG-06927, EY-07099, and AR-39750 from the National Institutes of Health, by a grant from the American Federation for Aging Research, and by the Lester Conrad Foundation.

\section{References}

1. Monnier, V. M., V. Vishwanath, K. E. Frank, C. A. Elmets, P. Dauchot, and R. R. Kohn. 1986. Relation between complications of type I diabetes mellitus and collagen-linked fluorescence. $N$. Engl. J. Med. 314:403-408.

2. Sell, D. R., and V. M. Monnier. 1989. Isolation, purification and partial characterization of novel fluorophores from aging human insoluble collagen-rich tissue. Connect. Tissue Res. 19:77-92.

3. Sell, D. R., and V. M. Monnier. 1989. Structure elucidation of a senescence crosslink from human extracellular matrix: implication of ribose in the aging process. J. Biol. Chem. 264:21597-21602.

4. Heaf, D. J., and J. I. Davies. 1976. The effect of RNA supplementation of rat diets on the composition of body fluids. Br. J. Nutr. 36:381-402.

5. Wyngaarden, J. B., S. Segal, and J. Foley. 1957. Physiological disposition and metabolic fate of infused pentoses in man. J. Clin. Invest. 36:1395-1407.

6. Segal, S., and J. Foley. 1958. The metabolism of D-ribose in man. J. Clin. Invest. 37:719-735.

7. Stegeman, H., and S. Stalder. 1967. Determination of hydroxyproline. Clin. Chim. Acta. 18:267-273.

8. Hamlin, C. R., and R. R. Kohn. 1971. Evidence for progressive age-related structural changes in post-mature human collagen. Biochim. Biophys. Acta. 236:458-467.

9. Shapiro, S. S., and M. B. Wilk. 1965. An analysis of variance test for normality. Biometrika. 52:591-611.

10. Steel, R. G. D., and J. H. Torrie. 1980. Principles and Procedures of Statistics. McGraw-Hill Inc., New York. 471, 542-543.

11. Neter, J., and W. Wasserman. 1974. Applied Linear Statistical Models. Richard D. Irwin, Inc., Homewood, IL. 21-393.

12. Norusis, M. J. 1986. SPSS/PC+ for the IBM PC/XT/AT. SPSS, Inc., Chicago, IL. B197-B243.

13. Armitage, P. 1971. Statistical Methods in Medical Research. John Wiley \& Sons, New York. 163-165.

14. Bell, D. J., and Q.-K. Talukder. 1972. Rates of urinary excretion of free aldopentoses and fucose by fasting healthy adults: effects of ingested pentose-containing foods. Clin. Chim. Acta. 40:13-30.

15. Date, J. W. 1958. Quantitative determination of some carbohydrates in normal urine. Scand. J. Clin. Lab. Invest. 10:155-162.

16. Jolley, A. B., K. S. Warren, C. D. Scott, J. L. Jainchill, and M. L. Freeman. 1970. Carbohydrates in normal urine and blood serum as determined by high-resolution column chromatography. J. Am. Clin. Pathol. 53:793-802.

17. Brownlee, M., A. Cerami, and H. Vlassara. 1988. Advanced products of nonenzymatic glycosylation and the pathogenesis of diabetic vascular disease. Diabetes Metab. Rev. 4:437-451.

18. Velez, R. L., and W. L. Henrich. 1984. The vasculature. In The Systemic Consequences of Renal Failure. G. Eknoyan and J. P. Knochel, Editors. Grune \& Stratton Inc., Orlando, FL. 93-112.

19. Gilchrest, B. A., J. W. Rowe, and M. C. Mihm, Jr. 1980. Clinical and histologic cutaneous findings in chronic renal failure: evidence for a dialysis-resistant transplant responsive microangiopathy. Lancet. ii: $1271-1275$.

20. Touraine, J. L., F. Tourane, and J. P. Revilland. 1975. T-lymphocytes and serum inhibitors of cell mediated immunity in renal insufficiency. Nephron. 14:195-201.

21. Zier, K. S., M. M. M. Leo, R. S. Spielman, and L. Baker. 1984. Decreased synthesis of interleukin II (IL-2) in insulin-dependent diabetes mellitus. Diabetes. 33:552-555.

22. Eknoyan, G., and J. P. Knochel. 1984. The Systemic Consequences of Renal Failure. Grune \& Stratton Inc., Orlando, FL. 1-587.

23. Bergstrom, J., and P. Furst. 1976. Uremic middle molecules. Clin. Nephrol. 5:143-152.

24. Bergstrom, J., P. Furst, and L. Zimmerman. 1979. Uremic middle molecules exist and are biologically active. Clin. Nephrol. 11:229-238.

25. National Diabetes Data Group. 1979. Classifications and diagnosis of diabetes mellitus and other categories of glucose intolerance. Diabetes. 28:1039-1057. 